\title{
Leçons tirées du lancement du programme de naloxone à emporter à domicile du Manitoba
}

\author{
Songul Bozat-Emre, Ph. D. (1,2); Shelley G. Marshall, M. Sc. (3); Colin Zhong (1,4); Joss Reimer, M.D. (1,2,3)
}

Diffuser cet article sur Twitter

\begin{abstract}
Résumé
Le gouvernement du Manitoba a lancé son programme de naloxone à emporter à domicile en janvier 2017. Fin septembre 2017, plus de 60 sites de distribution fonctionnaient dans la province. Ces sites ont distribué 765 trousses aux personnes à risque de surdose d'opiö̈des, dont 93 en remplacement d'une trousse utilisée lors d'une surdose. La plupart de ces surdoses ont touché des hommes $(60,2 \%)$ et ont eu lieu dans une résidence privée $(72,0 \%)$. Le fentanyl et le carfentanil ont été les substances en cause les plus fréquemment rapportées dans les cas de surdose. Les données du programme de naloxone à emporter à domicile fournissent des renseignements importants sur le contexte spécifique de la crise des opioïdes au Manitoba.
\end{abstract}

Mots-clés : distribution de naloxone, opiö̈des, réaction à une surdose

\section{Introduction}

Les méfaits associés à la surdose et à l’abus d'opioïdes constituent un problème de santé publique grandissant au Manitoba comme dans les autres provinces canadiennes. Ne serait-ce qu'au Manitoba, le nombre de décès semblant liés aux opioïdes a augmenté de 87,5 \% entre le premier trimestre $2016(\mathrm{n}=16)$ et le premier semestre $2017(\mathrm{n}=30)^{1}$. On a constaté des changements importants dans les décès liés au fentanyl en 2017 dans la province, avec en particulier la détection de l'analogue carfentanil chez $40 \%$ des personnes décédées $(n=12)$. Cette tendance est préoccupante, car on considère que le carfentanil est de 50 à 100 fois plus puissant que le fentanyl, et une dose ne serait-ce que d'un microgramme peut avoir des effets toxiques chez les humains ${ }^{2}$.

En riposte au début de la crise des opioïdes, le programme Connexions Street de l'Office régional de la santé de Winnipeg a lancé en janvier 2016 le premier programme de naloxone à emporter à domicile du Manitoba. Ses composantes clés ont été créées à partir du Programme de la naloxone à emporter à domicile du BC Centre for Disease Control [centre de lutte contre la maladie de la Colombie-Britannique $]^{3}$, puis enrichies par les apports d'une consultation qualitative auprès des usagers d'opioïdes à Winnipeg et d'autres intervenants clés.

Le ministère de la Santé, des Aînés et de la Vie active (MSAVA) du Manitoba a adapté le programme pilote de Winnipeg afin de lancer une initiative à l'échelle provinciale en janvier 2017, dans le cadre d'un plan d'intervention en matière de santé publique pour les opioïdes : il fournit gratuitement des trousses de naloxone à emporter aux personnes à risque de surdose d'opioïdes, en offrant une formation sur la prévention et l'identification des surdoses et les mesures à prendre dans ce cas, en particulier l'administration de naloxone. Une liste des composantes de la trousse de naloxone à emporter, les critères des sites de

\section{Points saillants}

- Les programmes de naloxone à emporter à domicile doivent être conçus de manière à réduire au minimum le fardeau des ressources humaines associé à la distribution, ce qui en facilitera l'accès.

- Les indicateurs du programme de naloxone à emporter à domicile peuvent fournir des renseignements importants sur la dynamique du marché des drogues et les méfaits qui lui sont associés.

distribution et un manuel de formation sont disponible en ligne à partir de la page http://www.gov.mb.ca/fentanyl/index.fr .html. Une liste à jour des sites de distribution des trousses de naloxone à emporter au Manitoba est disponible sur la page http://www.streetconnections.ca/?form _name = filter_language\&form_type $=$ filter\&language_id $=2$.

Dans cet article, nous présentons les principaux résultats de l'évaluation du programme de naloxone à emporter du Manitoba entre le $1^{\text {er }}$ janvier et le 30 septembre 2017, ainsi que certaines des principales fonctions du programme qui en ont permis l'expansion rapide.

\section{Méthodologie}

L'évaluation du programme de naloxone à emporter repose sur trois sources principales de données. Premièrement, les sites officiels de distribution de naloxone à 
emporter ont été inscrits dans un répertoire créé dans Panorama (un système électronique de gestion de la santé publique), avec des renseignements sur le nombre de sites et sur le nombre de trousses de naloxone à emporter livrées sur chaque site par l'entrepôt provincial de naloxone. Deuxièmement, la Division de la santé publique du MSAVA a demandé aux sites de distribution de lui indiquer le nombre de trousses distribuées aux personnes à risque de surdose d'opioïdes (formulaire PDF disponible en ligne à https://www.gov.mb.ca/health/publichealth /surveillance/docs/mhsu_6259_20171115 .pdf). Troisièmement, lorsqu'un intervenant profane utilise une trousse en cas de surdose, le membre du personnel qui fournit une trousse de remplacement est tenu de compléter un formulaire de réponse à une surdose (disponible en ligne à https://www .gov.mb.ca/health/publichealth/surveillance /docs/mhsu_6836_20161215.pdf) afin d'as surer la surveillance et l'évaluation du programme. Nous avons effectué l'analyse des données tirées de ces trois sources pour la période du $1^{\text {er }}$ janvier au 30 septembre 2017.

\section{Résultats}

Au Manitoba, neuf sites de distribution étaient actifs lors du lancement du programme provincial de naloxone à emporter et plus de 60 sites de distribution l'étaient en septembre 2017, dont 24 au sein des collectivités des Premières Nations. Entre le $1^{\text {er }}$ janvier et le 30 septembre 2017, l'entrepôt provincial de naloxone a livré 1360 trousses de naloxone à emporter aux divers sites de distribution du Manitoba et ces sites ont distribué 765 trousses à des personnes à risque de surdose d'opioïdes. Parmi ces 765 trousses, 93 étaient des trousses de remplacement pour une trousse utilisée dans la collectivité lors d'une surdose, ce qui indique qu'environ une trousse sur huit est utilisée lors d'un cas présumé de surdose d'opioïdes.

Une grande proportion de ces 93 surdoses présumées d'opioïdes (où des trousses de naloxone ont été utilisées) a touché des personnes de 12 à 30 ans (48,3 \%) (tableau 1). De plus, 56 étaient des hommes, 30 étaient des femmes et les 7 autres étaient des personnes de sexe inconnu ou ayant préféré ne pas répondre. La plupart ont eu lieu dans la région sanitaire de Winnipeg $(79,6 \%)$ et en résidence privée $(72,0 \%)$. Les comprimés buvards de fentanyl $(36,6 \%)$ et de carfentanil $(23,7 \%)$ de contrebande ont été les drogues les plus

TABLEAU 1

Caractéristiques des 93 cas présumés de surdose d'opioïdes avec utilisation d'une trousse de naloxone à emporter, Manitoba, du $1^{\mathrm{er}}$ janvier au 30 septembre 2017

\begin{tabular}{|c|c|c|}
\hline Caractéristiques & Catégories & n (\%) \\
\hline \multirow[t]{3}{*}{ Sexe } & Femme & $30(32,3)$ \\
\hline & Homme & $56(60,2)$ \\
\hline & Inconnu/Préfère ne pas répondre & $7(7,5)$ \\
\hline \multirow[t]{4}{*}{ Groupe d'âge (ans) } & 12 à 30 & $45(48,3)$ \\
\hline & 31 à 40 & $23(24,7)$ \\
\hline & 41 ou plus & $12(13,0)$ \\
\hline & Inconnu/préfère ne pas répondre & $13(14,0)$ \\
\hline \multirow[t]{3}{*}{ Lieu de survenue de la surdose } & Résidence privée & $67(72,0)$ \\
\hline & Rue & $7(7,5)$ \\
\hline & Autrea/Préfère ne pas répondre & $19(20,5)$ \\
\hline \multirow{3}{*}{$\begin{array}{l}\text { Région sanitaire de survenue de la } \\
\text { surdose }\end{array}$} & Winnipeg & $74(79,6)$ \\
\hline & Autres régions sanitaires & $13(13,9)$ \\
\hline & $\begin{array}{l}\text { À l'extérieur de la province/Inconnue/ } \\
\text { Préfère ne pas répondre }\end{array}$ & $6(6,5)$ \\
\hline \multirow[t]{5}{*}{ Type de substance ${ }^{b}$} & Fentanyl & $34(36,6)$ \\
\hline & Carfentanil & $22(23,7)$ \\
\hline & Méthamphétamine en cristaux & $13(14,0)$ \\
\hline & Morphine & $9(9,7)$ \\
\hline & Autres substances ${ }^{c}$ & $15(16,0)$ \\
\hline
\end{tabular}

${ }^{a}$ Les autres lieux sont des toilettes publiques, un hôtel, un refuge et un véhicule.

${ }^{\mathrm{b}}$ Les résultats ne sont pas mutuellement exclusifs.

' Les autres substances sont les benzodiazépines, la cocaïne et le crack, l’alcool, la codéine, la méthadone, l’héroïne et le dilaudid.

souvent déclarées dans les cas de surdose d'opioïdes soupçonnée où des trousses de naloxone ont été utilisées.

Dans 49 des 93 cas déclarés de surdose (52,6 \%), le répondant profane n’a pas appelé le 911 (tableau 2). Les principales raisons invoquées ont été : «je pensais que l'état de la personne s'améliorerait de luimême » $(22,5 \%)$, « je craignais l'intervention de la police » $(20,4 \%)$ et « il n'y avait pas de téléphone »(14,3\%). Les principales mesures prises par le répondant profane dans les cas de surdose ont été : " vérifier la respiration de la personne " $(60,2 \%)$ et « pratiquer la respiration artificielle » $(41,9 \%)$. De plus, la moitié des femmes en surdose ont reçu une seule dose (soit $0,4 \mathrm{mg}$ ) de naloxone, tandis que les hommes en surdose en ont le plus souvent reçu deux (53,6 \% données non présentées).

\section{Analyse}

Le Manitoba a réussi à mettre en œuvre son programme de naloxone à emporter sur une période relativement courte.
L'expansion rapide de ce programme est illustrée par la croissance du nombre et de la couverture des sites de distribution, avec des sites actifs au cours des neuf premiers mois de fonctionnement dans la plupart des régions sanitaires et dans plus de 24 collectivités des Premières Nations.

Cette réussite est attribuable à plusieurs facteurs, à la fois contextuels et spécifiques au programme. Tout d'abord, les premiers programmes de naloxone à emporter réalisés dans d'autres provinces, programmes ou régions avaient déjà prouvé leur efficacité $^{4}$. Ce corpus de données a soutenu l'expansion de la distribution de naloxone à emporter, en particulier au Manitoba.

Deuxièmement, le report de l'inscription de la naloxone sur la liste des drogues sur ordonnance de Santé Canada ${ }^{5}$ en 2016 a permis au Manitoba de lancer son programme en 2017 avec des critères relatifs aux sites de distribution moins restrictifs, car la présence d'un médecin prescripteur n'était pas obligatoire. De plus, le programme 
TABLEAU 2

Caractéristiques des 93 interventions d'urgence lors d'une surdose soupçonnée d'opioïdes avec utilisation d'une trousse de naloxone à emporter, Manitoba, du $1^{\text {er }}$ janvier au 30 septembre 2017

\begin{tabular}{|c|c|c|}
\hline Caractéristiques & Catégories & n (\%) \\
\hline \multirow{3}{*}{$\begin{array}{l}\text { Quelqu'un a-t-il appelé le } \\
\text { 911? }\end{array}$} & Oui & $34(36,6)$ \\
\hline & Non & $49(52,6)$ \\
\hline & Inconnue/préfère ne pas répondre & $10(10,8)$ \\
\hline \multirow{5}{*}{$\begin{array}{l}\text { Raison(s) de NE PAS } \\
\text { AVOIR APPELÉ le } 911^{a}\end{array}$} & Pas de téléphone & $7(14,3)$ \\
\hline & Craignait l'intervention de la police & $10(20,4)$ \\
\hline & $\begin{array}{l}\text { Pensait que l'état de la personne s'améliorerait de } \\
\text { lui-même }\end{array}$ & $11(22,5)$ \\
\hline & Autres raisons ${ }^{b}$ & $6(12,2)$ \\
\hline & Inconnue/préfère ne pas répondre & $15(30,6)$ \\
\hline \multirow{13}{*}{$\begin{array}{l}\text { Mesures prises pendant la } \\
\text { surdose }^{\mathrm{a}}\end{array}$} & Est resté avec la personne jusqu'à ce qu'elle revienne à elle & $54(58,1)$ \\
\hline & A vérifié la respiration de la personne & $56(60,2)$ \\
\hline & A pratiqué la respiration artificielle & $39(41,9)$ \\
\hline & A giflé ou secoué la personne (non recommandé) & $34(36,6)$ \\
\hline & A placé la victime en position de récupération & $25(26,9)$ \\
\hline & A vérifié le pouls de la personne & $32(34,4)$ \\
\hline & A crié après la personne & $39(41,9)$ \\
\hline & A pratiqué des compressions thoraciques & $20(21,5)$ \\
\hline & $\begin{array}{l}\text { Est resté avec la personne jusqu'à l'arrivée des premiers } \\
\text { secours }\end{array}$ & $28(30,1)$ \\
\hline & A vérifié les voies respiratoires de la personne & $20(21,5)$ \\
\hline & A donné à la personne un massage thoracique & $30(32,3)$ \\
\hline & Autres mesures prises ${ }^{c}$ & - \\
\hline & Inconnue & $17(18,3)$ \\
\hline \multirow{4}{*}{$\begin{array}{l}\text { Nombre de doses de } \\
\text { naloxone }^{\text {d données }}\end{array}$} & Une & $30(32,3)$ \\
\hline & Deux & $40(43,0)$ \\
\hline & Trois & $12(12,9)$ \\
\hline & Nombre inconnu & $11(11,8)$ \\
\hline
\end{tabular}

${ }^{a}$ Les résultats ne sont pas mutuellement exclusifs.

${ }^{\text {b } L e s ~ a u t r e s ~ r a i s o n s ~ s o n t ~ l e ~ f a i t ~ q u e ~ l a ~ p e r s o n n e ~ e n ~ s u r d o s e ~ a ~ d e m a n d e ́ ~ d e ~ n e ~ p a s ~ a p p e l e r ~ l e ~ 911, ~ q u ' e l l e ~ a ~ e ́ t e ́ ~ t r a n s p o r t e ́ e ~ a ̀ ~}$ l'urgence par l'entourage ou qu'elle s'est remise rapidement.

' Les autres mesures prises pendant la surdose sont le fait de donner une douche froide à la personne ou de la stimuler avec de la glace.

${ }^{d}$ Une dose de naloxone est de 0,4 mg (c.-̀̀-d. $0,4 \mathrm{mg} / \mathrm{ml}$ ).

— : Données supprimées en raison de la petite taille de l'échantillon (c.à-d. $1 \leq n \leq 5$ ).

a offert une formation en cas de surdose à toute personne souhaitant devenir répondant profane en cas de surdose, ce qui a réduit le fardeau des ressources humaines sur les sites de distribution pour les fournisseurs de soins de santé.

Enfin, le MSAVA a établi des partenariats clés avant le renforcement des critères relatifs aux sites de distribution pour s'assurer que le programme était suffisamment souple pour être déployé dans des milieux urbains et éloignés variés. Les consultations avec la Direction générale de la santé des Premières nations et des Inuits et les conseils tribaux régionaux ont joué un rôle clé et une entente de recouvrement des coûts a été conclue. Cette démarche a permis aux collectivités des Premières Nations et d'autres collectivités d'avoir un accès identique aux trousses de naloxone à emporter et a permis en outre de promouvoir une formation normalisée à l'échelle de la province et d'établir un mécanisme de collecte et de partage des données sur la distribution de naloxone qui respecte les principes de propriété, de contrôle, d'accès et de possession des Premières Nations ${ }^{6}$.

Nos données mettent en évidence une réticence persistante des répondants profanes à appeler le 911 en cas de surdose en raison de la crainte d'être arrêtés ou malmenés par la police. La Loi sur les bons samaritains secourant les victimes de surdose, promulguée en mai 2017, accorde l'immunité d'arrestation pour possession simple de drogue à une personne qui appelle le 911 en cas d'urgence. Les données du programme de naloxone vont permettre d'évaluer l'incidence de ce changement de politique sur la volonté des répondants profanes à composer le 911 en situation de surdose.

Nous avons constaté, d'après les données sur les trousses de remplacement, que les comprimés buvards de fentanyl et de carfentanil de contrebande étaient les drogues les plus souvent déclarées dans les cas de soupçon de surdose d'opioïdes. Les autres centres urbains du Canada ne semblent pas avoir signalé cette forme de commercialisation et de trafic du fentanyl et du carfentanil de contrebande. La présence de ces produits laisse penser à une recherche et une consommation intentionnelles de ces drogues, mais avec un dosage non sécuritaire. Cette information est issue des rapports subjectifs sur les drogues que les propriétaires de la trousse de naloxone ont fournis et repose sur leurs hypothèses sur l'origine de la surdose. Bien que ces réponses n'aient pas validées été (et ne puissent pas l'être) par une vérification des drogues ou par des analyses toxicologiques, elles constituent une source d'information très utile sur les marchés de drogues locaux.

\section{Conclusion}

Le Manitoba a mis en œuvre avec succès un programme provincial de naloxone à emporter sur une période relativement courte, ce qui lui permet de prévenir les surdoses d'opioïdes et d'en réduire les méfaits en situation d'urgence. Les données dérivées de ce programme fournissent des renseignements importants sur le contexte particulier de l'utilisation des opioïdes et des méfaits qui lui sont associés au Manitoba, elles enrichissent les rapports de 
surveillance des opioïdes et elles peuvent éclairer d'autres interventions en santé publique. Les programmes de distribution des trousses de naloxone dépendent finalement de l'expertise des personnes ayant un vécu expérientiel et offrent ainsi des possibilités de réelle collaboration entre les fournisseurs de services de santé et les personnes à risque de surdose d'opioïdes.

\section{Conflits d'intérêts}

Les auteurs n'ont aucun conflit d'intérêts à déclarer.

\section{Contributions des auteurs et avis}

SBE a interprété les données et rédigé et revu le manuscrit; SM a conçu et conceptualisé le travail, a analysé et interprété les données et a rédigé et révisé le manuscrit; CZ a analysé les données et révisé le manuscrit; JR a conceptualisé l'étude et révisé le manuscrit.

Le contenu de cet article et les opinions qui y sont exprimées n'engagent que les auteurs et ne représentent pas forcément les opinions du Gouvernement du Canada.

\section{Références}

1. Gouvernement du Manitoba, ministère de la Santé, des Aînés et de la Vie active du Manitoba, Division de la santé publique et des soins primaires, Épidémiologie et surveillance. Surveillance of opioid misuse and overdose in Manitoba: April 1 - June 30, 2017. Winnipeg (Man.) : Gouvernement du Manitoba; 2017. En ligne à : http:// www.gov.mb.ca/health/publichealth /surveillance/docs/q2_opioid.pdf

2. Raffa RB, Pergolizzi JV, LeQuang JA, Taylor R, Colucci S, Annabi MH. The fentanyl family: a distinguished medical history tainted by abuse. J Clin Pharm Ther. 2018;43(1):154-158.

3. BC Centre for Disease Control. IBC's Take Home Naloxone Program [Internet]. Vancouver (C.-B.) : BCCDC; 2012 [consulté le 12 janvier 2017]. En ligne à : http://towardtheheart.com/ezine /3/take-home-naloxone-program

4. Clark AK, Wilder CM, Winstanley EL. A systematic review of community opioid overdose prevention and naloxone distribution programs. J Addict Med. 2014;8(3):153-163.
5. Santé Canada. Avis : Liste des drogues sur ordonnance (LDO) : Naloxone [Internet]. Ottawa (Ont.) : Santé Canada; [modifié le 22 mars 2016, consulté le 12 janvier 2017]. En ligne à : https://www.canada.ca/fr/sante - canada/services/medicaments -produits-sante/medicaments/liste -drogues-ordonnance/notice-naloxone .html

6. Centre de gouvernance de l'information des Premières Nations. Les principes PCAP des Premières Nations [Internet]. Akwesasne (Ont.) : Centre de gouvernance de l'information des Premières Nations; [mis à jour en 2018; consulté le 12 janvier 2017]. En ligne à : http://fnigc.ca/fr/pcap.html 